\title{
Bone morbidity in pediatric acute lymphoblastic leukemia
}

\author{
Moon Bae Ahn, MD, \\ Byung-Kyu Suh, MD, PhD
}

Department of Pediatrics, College of Medicine, Catholic University of Korea, Seoul, Korea
Received: 28 January, 2020

Revised: 6 March, 2020

Accepted: 12 March, 2020

Address for correspondence:

Byung-Kyu Suh, MD, PhD

Department of Pediatrics, College of

Medicine, The Catholic University of

Korea, 222 Banpo-daero, Seocho-gu,

Seoul 06591, Korea

Tel: +82-2-2258-6756

Fax: +82-2-537-4544

E-mail:suhbk@catholic.ac.kr

https://orcid.org/0000-0003-19710232
Acute lymphoblastic leukemia (ALL), currently the most common pediatric leukemia, has a high curability rate of up to $90 \%$. Endocrine disorders are highly prevalent in children with ALL, and skeletal morbidity is a major issue induced by multiple factors associated with ALL. Leukemia itself is a predominant risk factor for decreased bone formation, and major bone destruction occurs secondary to chemotherapeutic agents. Glucocorticoids are cornerstone drugs used throughout the course of ALL treatment that exert significant effects on demineralization and osteoclastogenesis. After completion of treatment, ALL survivors are prone to multiple hormone deficiencies that eventually affect bone mineral accrual. Dualenergy X-ray absorptiometry, the most widely used method of measuring bone mineral density, is used to determine the presence of childhood osteoporosis and vertebral fracture. Supplementation with calcium and vitamin D, administration of pyrophosphate analogues, and promotion of mobility and exercise are effective options to prevent further bone resorption and fracture incidence. This review focuses on addressing bone morbidity after pediatric ALL treatment and provides an overview of bone pathology based on skeletal outcomes to increase awareness among pediatric hemato-oncologists and endocrinologists.

Keywords: Acute lymphoblastic leukemia, Bone mineral density, Vertebral fracture, Osteoporosis

\section{Introduction}

Acute lymphoblastic leukemia (ALL) is the most common cancer among pediatric and adolescent patients, and it accounts for major cancer-related deaths in childhood. ${ }^{1)}$ Various factors, including exogenous (e.g., infection) or endogenous exposures (e.g., inflammation, oxidative stress) and genetic background, contribute to disease onset; ALL occurs in roughly 1:2,000 children aged $<15$ years. ${ }^{2)}$ After successful evolution of chemotherapy and clinical trials spanning 5 decades, the current curability rate of ALL is approximately $90 \%$ in resource-rich countries. ${ }^{3)}$

Endocrine disorders are highly prevalent among cancer survivors; recent data indicate that $40 \%-50 \%$ of survivors will develop at least one endocrinopathy once in their lifetime. ${ }^{4)}$ Endocrine dysfunction is encountered in pediatric patients with ALL during and after therapy and might arise in the long term. Endocrine complications in children during and after ALL treatment include compromised growth, hyperglycemia, thyroid dysfunction, adrenocortical insufficiency, gonadal dysfunction, syndrome of inappropriate secretion of antidiuretic hormone, and bone demineralization. ${ }^{5)}$

Growing bone is vulnerable to the leukemic process and chemotherapeutic drugs. ALL is a classic malignancy for developing skeletal morbidities ${ }^{6}$ such as pain, fractures, decreased bone mineral density (BMD), and chronic impairment of bone function, which may be present not only during ALL diagnosis, but also as long-term sequelae following therapy. ${ }^{7)}$ Osteoporosis 
originating from ALL is triggered by multiple factors, including leukemic infiltration of bone, reduced bone strength due to immobility, poor growth induced by nutritional deficiency, and the use of various osteotoxic drugs. ${ }^{8)}$ This review addresses bone morbidity after pediatric ALL treatment and provides an overview of bone pathology based on skeletal outcomes to increase awareness among pediatric hemato-oncologists and endocrinologists.

\section{Bone morbidity at ALL diagnosis}

Lengthwise bone growth in children with ALL is not different from that in healthy children, whereas bone density is reduced by lower bone turnover. ${ }^{9)}$ Defective mineralization and reduced bone formation are recognized in most children with ALL prior to treatment initiation. ${ }^{9)}$ Cytokines released by leukemic cells trigger osteoclast-mediated bone resorption, which causes bone pain and osteopenia, and bone turnover marker levels are diminished before treatment. ${ }^{10)}$ This indicates that the leukemic process itself is a predominant risk factor for decreased bone formation.

Juxta-metaphyseal lucent bands and osteolytic lesions on plain radiography are present in $70 \%$ of ALL cases at initial diagnosis and occur as part of the leukemic process. ${ }^{11)}$ Tumorassociated factors upregulate receptor activator of nuclear factor kappa- $\beta$ ligand, resulting in activation of osteoclasts, triggering of osteolytic lesions, and destruction of endochondrons. ${ }^{12)}$ Bone pain is a common presenting symptom of childhood ALL that occurs in over $40 \%$ of cases, and it is the most common sign of undermineralized bone. Bone pain mainly occurs due to uncontrolled osteoclastic activity causing altered sympathetic nerve fiber regulation and neuropeptide release. ${ }^{13,14)}$ Periosteal reaction $(<19 \%)$, low bone mass $(<40 \%)$, and fractures $(<10 \%)$ are consecutive results due to infiltration of leukemic cells in bone. ${ }^{9)}$

\section{Definition of low bone mass}

The definition of osteoporosis remains too controversial to be applied to children and adolescents, and densitometric outcome does not fully confirm diagnosis. The International Society of Clinical Densitometry (ISCD) defines osteoporotic children based on the presence of fracture and lowered densitometric standard deviation score for age and sex. The ISCD definition for osteoporosis in children is based on a clinically significant fracture history and a BMD $z$-score $\leq-2.0$ (Table 1) at preferred

Table 1. Considerations for the assessment of skeletal health in children and adolescents

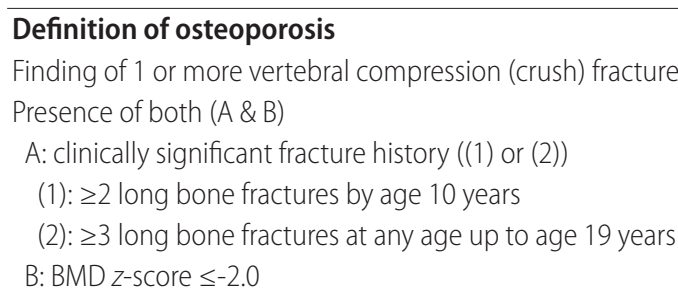

\section{DXA assessment (BMC and aBMD)}

Should be performed when the patient may benefit from interventions to decrease their elevated risk of a clinically significant fracture and the results will influence management

Should not be performed if safe and appropriate positioning of the child cannot be assured

Preferred site:

Poster-anterior spine and total body less head

Feasible sites of measurements:

Lumbar spine for $0-5$ years of age

Whole-body for $\geq 3$ years of age

\section{DXA interpretation and reporting}

Adequate reference data should be available

Additional possible sites: proximal femur, 33\% radius (1/3 radius), lateral distal femur

Minimum interval for follow-up is 6-12 months

For those who have short stature or growth delay, results should be adjusted based on height z-score

\section{VF assessment}

Genant semiquantitative method should be used

Following VF assessment, additional spine imaging such as quantitative computed tomography should be considered in the following circumstances: Vertebra that are technically un-evaluable (not sufficiently visible)

A single, Genant grade $1 \mathrm{VF}$

Radiographic findings that are not typical for an osteoporotic VF (suspected destructive inflammatory or malignant processes, congenital malformations, acquired misalignments, or dislocations)

BMD, bone mineral density; DXA, dual-energy x-ray absorptiometry; BMC, bone mineral content; aBMD, areal bone mineral density; VF, vertebral fracture; z-score, standard deviation score. 
sites. ${ }^{15)}$ A clinically significant fracture history indicates 1 or more of the following: ( 1$) \geq 2$ long bone fractures by age 10 years or (2) $\geq 3$ long bone fractures at any age up to 19 years. ${ }^{15)}$ The presence of $\geq 1$ nontraumatic vertebral compression (crush) fracture is indicative of osteoporosis in children. ${ }^{15)}$

\section{Nontraumatic vertebral fracture}

Nontraumatic vertebral fracture (VF) is a commonly encountered skeletal manifestation in children and adolescents with newly diagnosed ALL that occurs in as many as $16 \%$ of cases. ${ }^{9)}$ Cummings et al. reported a 4-year cumulative incidence of VF following ALL diagnosis of 26\%, with T5-7 as the most severely fractured levels. ${ }^{16)}$ Back pain can occur in $<55 \%$ of children at ALL diagnosis, and most fractures occur at thoracolumbar $(31 \%$, T12-L2) and midthoracic (24\%, T6-T7) levels. ${ }^{17)}$ Because most children do not manifest with back pain at initial presentation, this is under-recognized. ${ }^{18)}$ Moderate or severe VF can also occur asymptomatically; therefore, routine vertebral assessment as systematic surveillance on ALL diagnosis is fundamental. ${ }^{16)}$ VF can be diagnosed by conventional radiograph, and its assessment is useful as VF severity correlates with the degree of lumbar spine BMD.

\section{Imaging methods for evaluation of bone mass}

Conventional radiology, hand examination, and morphometric study of the spine are used to examine trabecular thickness and cortical thinning. ${ }^{19)}$ VF can be recognized by measuring the heights of vertebral bodies on a conventional radiograph. To visualize radiologic signs of reduction in bone density, a $30 \%-40 \%$ loss of mineralized bone is necessary. ${ }^{19)}$ In 1993, a "semiquantitative method" grading system was proposed to assess VF based on vertebral shape and reduction in height by qualitatively and quantitatively assessing osteoporotic vertebral deformity. ${ }^{20)}$ Depending on the degree of reduction in vertebral body height, grading reflects the severity of VF. Other scoring systems for assessment of VF through conventional radiography, such as an algorithm-based quantitative method, Koerber's technique, and a semiautomated technique, are used in clinical practice. ${ }^{21,22)}$ Yet, there is no reliable method available specifically designed for pediatric use that encompasses normal physiologic variation and observer variability. Thus, current techniques need further improvement to develop software tools to diagnose VF in the pediatric population. ${ }^{23)}$

Quantitative computed tomography (QCT) is beneficial because it measures bone volume and computes volumetric $\mathrm{BMD}$, unlike planar images generated by dual-energy X-ray absorptiometry (DXA); it also differentiates dense cortical bone from metabolically active trabecular bone. ${ }^{24)}$ With good accuracy and precision, QCT causes exposure to low radiation dose (approximately 3 microsievert per scan) and enables functional evaluation of pediatric bone disease. ${ }^{25)}$ QCT can measure both axial and appendicular sites, and a tabletop peripheral QCT device can measure the radius, tibia, or femur in children. ${ }^{26)}$ However, pitfalls of QCT include limited evaluation of appendicular bone with low turnover, low spatial resolution, and exact reposition of the extremity required for follow-up. ${ }^{25)}$

DXA remains the most commonly used imaging modality for measuring BMD because it confirms low bone mass. DXA determines the amount of bone mineral in a specific region by differential absorption of X-rays of 2 different energies and calculates the depth and composition of adjacent soft tissue to generate measurements of fat and lean mass. ${ }^{27)}$ Measurable sites in adults are the posterior-anterior spine and hip, and posterioranterior spine and total body without head are the preferred sites for performing bone mineral content and areal BMD (aBMD). ${ }^{15)}$ DXA only measures the 2 dimensional density of bone and not 3 dimensional volumetric density. ${ }^{28)}$ Hence, because the depth of bone is not considered, DXA is size-dependent and overestimates aBMD in large bones and underestimates aBMD in small bones. This is challenging for children in whom chronic disease stunts skeletal growth. ${ }^{29)}$ Furthermore, height and bone age-adjusted aBMD $z$-scores are more reliable values as they avoid pitfalls such as low aBMD $z$-scores due to short stature. ${ }^{30,31)}$ BMD differs according to age, sex, race, ethnicity, and lifestyle, requiring appropriate references for the pediatric population. Lim et al. ${ }^{31)}$ released BMD reference values in healthy Korean children and adolescents were released in 2010.

\section{Bone morbidity during leukemia treatment}

Administration of chemotherapeutic agents also destroys bone formation, and the adverse effects of therapy, rather than the antileukemia response, should be addressed during treatment. ALL treatment usually spans 3 years and comprises 3 phases of chemotherapy (remission-induction, consolidation, and maintenance), whereas hematopoietic stem cell transplantation (HSCT) with or without irradiation remains an option for patients at very high risk. ${ }^{2)}$ Among current regimens of chemotherapy against ALL, osteotoxic drugs such as glucocorticoids, methotrexate, L-asparaginase, daunorubicin, and vincristine, as well as irradiation treatment, are predominant risk factors that equally cause deficient BMD. The accumulated dosage and duration of therapy result in bone destruction (Table 2).

\section{Glucocorticoids}

Prolonged and repetitive high-dose glucocorticoids administered throughout treatment significantly contribute to bone morbidity. ${ }^{32)}$ Glucocorticoids (prednisolone and dexamethasone) are cornerstone drugs in first-line ALL treatment and may be used throughout the entire treatment period. Glucocorticoids are most highlighted during the remission-induction phase, when they have a strong cytotoxic effect on cancer cells by eliminating $99 \%$ of disease tissue. ${ }^{33)}$ During central nervous systemic-directed therapy, the success of ALL treatment depends on effective central nervous 
Table 2. Triggering factors of bone morbidity in children and adolescents with acute lymphoblastic leukemia

At ALL diagnosis
Back pain prior to VF screening
Reduced BMD
Low bone turnover markers
Infiltration of leukemic cells mediated cytokines
Age of ALL diagnosis near to peak bone mass period
During ALL treatment
Osteotoxic drugs
Glucocorticoids
Methotrexate
L-asparaginase
Daunorubicin
Vincristine
Irradiation
Malnutrition and lack of physical activity
ALL, acute lymphoblastic leukemia; BMD, bone mineral density; VF,
vertebral fracture.

systemic leukemia control, and glucocorticoids play an essential role along with prophylactic cranial irradiation. ${ }^{34)}$ In graftversus-host disease, an immune reaction occurring acutely or chronically after HSCT, glucocorticoids are used for both prophylaxis and treatment.

Glucocorticoid excess alters bone remodeling by increasing bone resorption and decreasing bone formation, leading to net loss of bone volume. ${ }^{35)}$ During treatment, glucocorticoids and multiple chemotherapeutic drugs suppress bone formation markers. Defective bone mineralization is affected by decreased production of serum calcitriol. ${ }^{9)}$ Glucocorticoids trigger hypercalciuria by increasing renal calcium excretion and decreasing intestinal calcium absorption and tubular reabsorption of phosphate, resulting in a negative calcium balance. ${ }^{36)}$ Osteocytes and osteoblasts are direct targets of glucocorticoid action via reduced osteoblast precursors combined with glucocorticoid-induced proapoptotic effects on osteoblasts. ${ }^{37)}$ Conversely, osteoclasts prolong their lifespan, and osteoclastogenesis is enhanced by glucocorticoid administration causing early and rapid bone loss. ${ }^{38,39)}$

Systemic glucocorticoid therapy is associated with an initial increase in bone resorption and subsequent reduced bone formation, resulting in microarchitectural deterioration and increased fracture risk. ${ }^{40)}$ Compared to cortical bone, trabecular bone in children is more sensitive to the detrimental effect of glucocorticoids. Duration and dosage of glucocorticoids negatively correlate with BMD, and the lumbar spine is the most affected location. ${ }^{39)}$ Bone loss assessed by DXA can be 5\%-15\% during the first year of treatment, and the major determinant of BMD is cumulative dose of glucocorticoids. ${ }^{41)}$ The greatest reduction of bone mineral content mostly occurs in the first 6-8 months of chemotherapy, although the chance of bone mass recovery depends on age and duration of glucocorticoid therapy. Pubertal children show significant decrements in spinal BMD compared to children who are prepubertal at diagnosis. ${ }^{42)}$ Prednisolone-equivalent corticosteroid doses in excess of $9 \mathrm{~g} / \mathrm{m}^{2}$ during ALL treatment pose a risk of deficits in BMD persisting into adulthood. ${ }^{43)}$

VFs are common manifestations occurring in 16\% of children at or within a month of ALL diagnosis, and incidental fractures peak at 12 months of treatment followed by a gradual decline in frequency. ${ }^{6}$ Nontraumatic VFs are asymptomatic; therefore, radiographic screening is the only method of surveillance. The presence of VF at ALL diagnosis is critical since the odds of an incidental fracture over the treatment period are associated with spinal BMD at baseline. ${ }^{44)}$ In one study evaluating a dexamethasone-based protocol, the 3-year cumulative fracture incidence was $18 \%$ in pediatric patients with ALL. ${ }^{45}$ Glucocorticoid exposure is a strong clinical predictor over the 4 -year incidence of VF, as incidence declines with a concurrent decrease in glucocorticoid exposure. ${ }^{16)} \mathrm{VF}$ can also lead to height loss in pediatric patients with ALL, and every $10-\mathrm{mg} / \mathrm{m}^{2}$ increase in average daily glucocorticoid dose is associated with height $z$-score decrement of $0.3 .^{46)}$

\section{Methotrexate}

By combining inhibition of purine and pyrimidine synthesis and reduced proliferation of antigen-dependent $\mathrm{T}$ cells, methotrexate has proven efficacious during all phases of ALL therapy. ${ }^{477}$ Methotrexate reduces bone mineralization by impairing osteoblast function, responsiveness, and number to suppress bone formation and subsequent osteopenia.9 Shortterm administration of therapeutic doses of methotrexate in animal studies caused a significant reduction in trabecular bone volume via cytotoxic effects on osteoblasts, whereas osteoclast activity was less disturbed. ${ }^{11)}$ Methotrexate is associated with reduced proliferation of preosteoblasts and osteoblasts, concurrently increasing osteoclast formation in the bone marrow and elevating osteoclast density on the bone surface, which eventually causes bone pain and fractures. ${ }^{43)}$ High-dose methotrexate in children with ALL decreases bone alkaline phosphatase and C-terminal propeptide of type I procollagen (marker of type I collagen synthesis), and increases C-terminal telopeptide of type I collagen (marker for breakdown of type I collagen). ${ }^{48}$ Despite long-term methotrexate therapy, discontinuation of the drug improves both clinical and radiographic manifestations of bone morbidity. ${ }^{49)}$

\section{Other chemotherapeutic drugs}

Other chemotherapeutic drugs, such as asparaginase and daunorubicin, contribute to reduced bone mineralization. $\mathrm{L}$-asparaginase converts $\mathrm{L}$-asparagine to aspartate and ammonia, thereby depleting serum L-asparagine and starving leukemic cells, leading to cell death. ${ }^{50)}$ Therapy with L-asparaginase increases plasma concentration of glucocorticoid (dexamethasone), leading to higher risk of osteonecrosis. ${ }^{51)}$ Daunorubicin, an anthracycline, is administered to patients at high risk. Daunorubicin prevents deoxyribonucleic acid replication via deoxyribonucleic acid 
topoisomerase II inhibition, deoxyribonucleic acid damage via formation of reactive oxygen species, and programmed cell death. ${ }^{52)}$ Anthracycline has deleterious effects on skeletal muscle tissue (muscle weakness, fatigue, and dysfunction) and causes reduced alkaline phosphatase activity in human osteoblastlike cells with $58 \%$ of control values, leading to adverse effects on bone mineral accrual. ${ }^{52,53)}$ Vincristine, a vinca alkaloid that disrupts microtubular formation, reduces type 1 collagen synthesis, induces neuropathy leading to poor balance, and increases the risk of falls. ${ }^{54)}$

\section{Irradiation}

Bony structures previously exposed to radiation are at risk for poor growth, and the risk is greater with higher radiation dosage and younger age at exposure. ${ }^{4)}$ Growth hormone $(\mathrm{GH})$ deficiency and hypogonadotropic hypogonadism induced by impaired hypothalamus-pituitary axis following cranial irradiation are associated with impaired BMD, whereas the direct osteotoxic effects of irradiation remain unclear. Decreased vertebral density was observed in patients with ALL who received cranial irradiation, albeit not in a dose-dependent manner. ${ }^{55)}$ Conversely, no significant differences in bone area and bone mineral content were observed between irradiated patients and non-irradiated patients. ${ }^{56)}$ Patients who received 24 Gy of cranial irradiation had low BMD, whereas patients who received $18 \mathrm{~Gy}$ had a BMD that did not differ from those who received no radiation therapy. ${ }^{57)}$ Therefore, deficient gonadal steroids, GH, and insulin-like growth factor (IGF)-1 from impaired hypothalamus-pituitary axis should be considered rather than the dosage or duration of cranial irradiation. The independent effects of chemotherapy should be elucidated to understand the direct effect of cranial irradiation on BMD.

\section{Malnutrition and lack of physical activity}

Optimization of nutrition is another critical factor of bone health during ALL treatment. Low serum calcitriol concentration after therapy completion correlates with reduced lumbar BMD regardless of normal calcium homeostasis. ${ }^{11)}$ Cachexia and being underweight result from poor caloric intake, nausea, emesis, and abdominal discomfort during chemotherapy followed by decreased bone formation. While high-dose glucocorticoid therapy leads to increased appetite during ALL treatment, a focused diet rich in only calcium and vitamin D is challenging in the pediatric age.

With adequate nutritional support, physical activity is an inevitable factor in BMD. During chemotherapy, overall physical activity diminishes; promotion of physical activity and exercise may ameliorate bone mass loss. ${ }^{9)}$ Exercise capacity and levels of physical activity were positively correlated with bone mineral content of the hip and spine. ${ }^{10)}$ Nevertheless, there are no recommendations for specific levels and intensity of physical activity and discriminating factors from usual care ${ }^{58)}$ During ALL treatment, children have reduced activity and diminished strength and fitness. Therefore, the clinical aim of physical activity via exercise is more focused on preventing bone fracture and osteoporosis than sustaining sufficient bone mineral accrual.

\section{Bone recovery after ALL treatment}

Despite direct leukemic effects and exposure to multiple osteotoxic treatment regimens, which altogether induce demineralization, the most rapid skeletal development occurs during childhood and adolescence. Skeletal recovery after therapy completion in children with ALL is crucial, while bone metabolic status continues to change significantly in this age group.

Survivors begin to recover lost bone mass after ALL therapy, while those who do not reach their optimal bone mineral acquisition experience critical bone loss 2 years following therapy cessation. ${ }^{7)}$ Associated clinical factors include glucocorticoid exposure and baseline lumbar spine BMD $z$-scores, which are both associated with VF and non-VF. ${ }^{59)}$ The BMD $z$-score for the lumbar spine and femoral neck reaches osteoporotic range 6 years after therapy; despite BMD increase after treatment completion, changes in $\mathrm{z}$-scores do not reveal full recovery. ${ }^{60)}$ Changes in cortical dimensions and BMD might depend on the interval after treatment cessation since early increase in cortical dimensions contributes to transient declines in cortical BMD, reflecting a longer time necessary to fully mineralize newly formed bone. ${ }^{61)}$ Yet, the extent of bone mass recovery in children after termination of therapy is unknown.

ALL survivors treated with cranial radiotherapy, craniospinal radiotherapy, and total body irradiation are prone to hypopituitary hormone deficiency and growth, and gonadal hormones predominantly act on bone mineral accrual. GH promotes bone formation via IGF-1 by stimulating osteoblastic activity. ${ }^{43)}$ Glucocorticoids alter bone health via decreasing the synthesis of IGF-1 and gonadotropins to result in deficiency of GH and sex steroid hormone. ${ }^{41,62)}$ Radiation directly affects the growth plate and causes GH deficiency and growth retardation. GH therapy is beneficial for BMD, bone geometry, and body composition and improves bone health. ${ }^{62)}$ Based on safety and efficacy, guidelines recommend GH replacement after a 1-year disease-free state in childhood cancer survivors with confirmed GH deficiency. ${ }^{4)}$ If not properly treated, primary or secondary hypogonadism compromises bone mineralization. Leydig cell failure is common if males are exposed to testicular radiation doses in excess of $20 \mathrm{~Gy}$, whereas females older than 10 years of age who receive $10 \mathrm{~Gy}$ of fractionated total body irradiation may experience ovarian failure. ${ }^{43)}$ Androgen receptors are located in growth plate osteoblasts and exert the anabolic effects of testosterone on bone, while estrogen has more direct activity in skeletal maturation and mineralization. ${ }^{63)}$ Compared to those who completed treatment with standard chemotherapy regimens, HSCT recipients are at higher risk of treatmentassociated GH deficiency and hypogonadism and subsequent poor bone acquisition. 
Vertebral reshaping is a growth-dependent bone remodeling phenomenon that involves acquiring normal vertebral dimensions following VF, which does not occur beyond epiphyseal fusion. ${ }^{6)}$ Seventy-seven percent of ALL children with VF had complete reshaping at the last follow-visit, $18 \%$ had incomplete reshaping, and 4\% were not reshaped over 6 years of follow-up. ${ }^{59)}$ Therefore, spontaneous vertebral reshaping is an opportunity for osteoporotic children to be conservatively observed, rather than to initiate pharmacologic intervention. ${ }^{64}$

\section{Management and recommendations}

Although many clinicians recommend calcium and vitamin D supplementation to remediate osteoporotic change, their role is controversial. ${ }^{65)}$ Calcium- and calcitriolretarded BMD loss in children receiving glucocorticoids was less in patients taking calcitriol and calcium compared to those taking placebo; this could be considered a preventive measure when supplementation begins simultaneously with glucocorticoid initiation. ${ }^{66)}$ Conversely, 2 years of vitamin D and calcium supplements in children with ALL after treatment completion did not significantly increase BMD compared to the placebo group. ${ }^{65)}$ There is no specific recommendation guideline for dosage of calcium and vitamin D supplements for children with ALL; therefore, $400 \mathrm{IU} /$ day of vitamin D and $500-1,300 \mathrm{mg} /$ day of calcium intake are recommended to prevent any deficiency. ${ }^{67,68)}$ Particularly, calcium and vitamin $\mathrm{D}$ should be adequate for effective pharmacologic treatment of osteoporosis since low calcium and cholecalciferol levels represent low bone mass. ${ }^{69)}$ Bone metabolic markers should be monitored throughout supplementation to avoid any episode of nephrolithiasis or urolithiasis from calcium excess.

Children with VF and/or low BMD and $\geq 2$ long bone fractures are considered for intravenous bisphosphonate (BP) therapy using potent antiresorptive agents, which have shown promising outcomes for treating VF in pediatric ALL patients (Fig. 1) ${ }^{6,8}$ BPs are synthetic analogues of pyrophosphate widely used in primary and secondary osteoporosis management. ${ }^{70)}$ BPs prevent osteoclastic bone resorption and relieve bone pain, while their use in prevention remains limited. ${ }^{6)}$ An Australian group recommends serum calcidiol level should be $>50 \mathrm{nmol} / \mathrm{L}$ prior to the first $\mathrm{BP}$ infusion and maintained postinfusion. ${ }^{8)}$ Increased $\mathrm{BMD}$, reduced bone pain, and improved vertebral body shape were observed after BP use in patients with hematologic malignancy and in bone marrow transplant patients with graft-versus-host disease, although there is limited case-control data. ${ }^{8)}$ Optimal agents, dose, and duration remain controversial, although intravenous agents may be more effective than oral agents. ${ }^{64)}$ Pamidronate $(9 \mathrm{mg} /$ $\mathrm{kg} / \mathrm{yr}, 4-6$ divided doses) and zolendronate $(0.05-0.1 \mathrm{mg} / \mathrm{kg} /$ yr, 2-4 divided doses) are widely used BPs. ${ }^{8,64)}$ Adverse effects are generally well-tolerated, although transient myalgia, bone pain, fever, and nausea are common after the first infusion. ${ }^{64)}$ BP therapy is the first-line treatment option for adults with low bone mass who receive glucocorticoid therapy for $>3$ months; however, there are insufficient data to support the efficacy of BPs in children exposed to excess glucocorticoids. ${ }^{39)}$ Although the long-term beneficial effects of BP therapy are currently unknown, it remains the optimal drug of choice for children with ALL since BPs can be used during steroid dosing and improve bone health despite other treatments.

Both calcitonin and teriparatide are effective at delaying the progression of osteoporosis. Calcitonin inhibits osteoclastic activity in bones, while teriparatide enhances bone formation. ${ }^{69,71)}$ However, the use of calcitonin and teriparatide in osteoporotic patients with ALL is limited, and their benefits compared to oral calcium and vitamin D or BP therapy are questionable.

Mobility and exercise during and after ALL treatment may be beneficial to bone mass acquisition. Exercise programs or physical activity interventions include exercises to maintain hand and leg function, stretching exercises to maintain ankle dorsiflexion mobility, and short-burst high-intensity exercises; outcomes of these programs revealed a significant positive

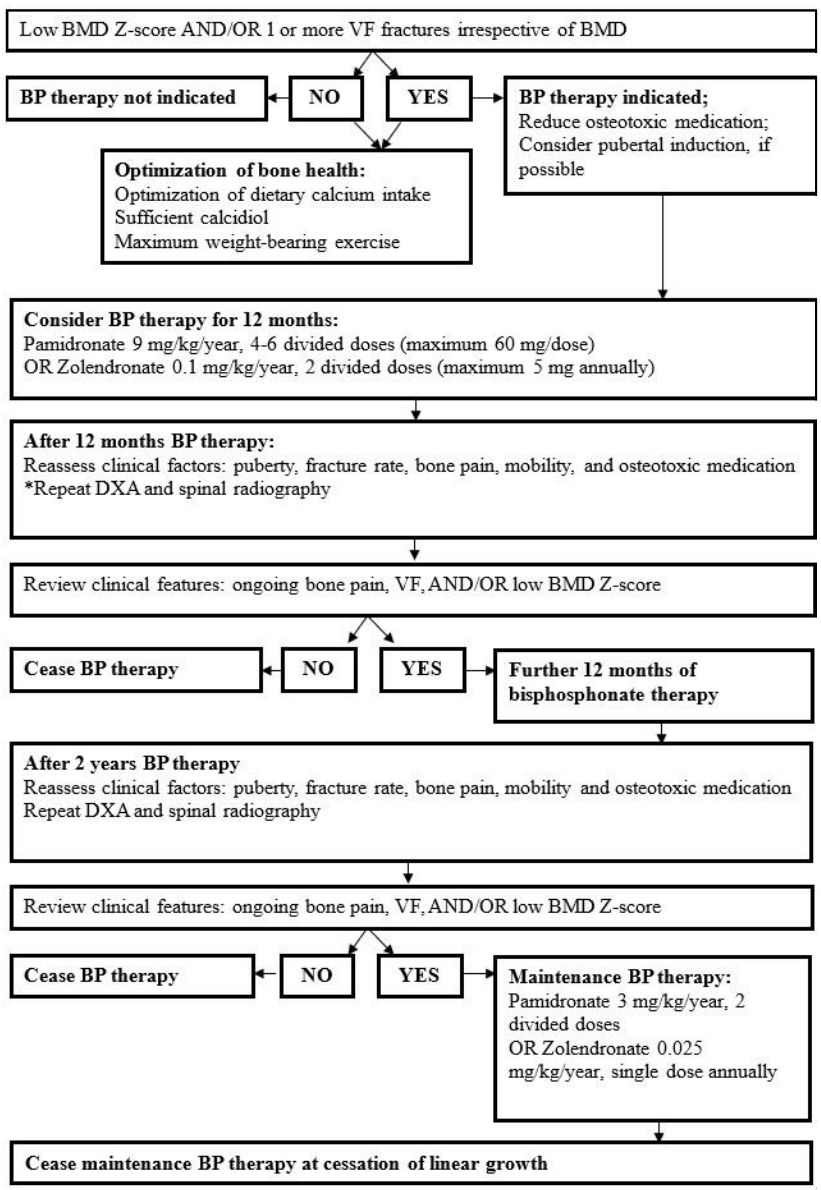

Fig. 1. Flow chart depicts the use of bisphosphonates and overall monitoring recommendations for children and adolescents with acute lymphoblastic leukemia induced osteoporosis. "Bi-annual dual-energy X-ray absorptiometry should be considered if factors associated with bone morbidity worsen. $B P$, bisphosphonate; $B M D$, bone mineral density; DXA, dual-energy $X$-ray absorptiometry, VF, vertebral fracture; $z$-score, standard deviation score. 
intervention effect on total body BMD. ${ }^{72)}$ Intrahospital supervised exercise may also considerably improve children's quality of life (QoL) and overall health status during treatment periods. ${ }^{73)}$

\section{Conclusion}

ALL is the most commonly encountered cancer during the pediatric and adolescent period, and bones are vulnerable to the leukemic process. Treatment of various skeletal complications diminishes QoL. From diagnosis until several years posttreatment, ALL may threaten bone health by triggering bone demineralization and reducing bone formation. Routine screening and monitoring of skeletal conditions via radiographic and laboratory assessment can lead to prompt recognition and intervention to prevent adverse long-term sequalae. Factors contributing to bone morbidity include chemotherapeutic drugs, irradiation, malnutrition, and lack of physical activity, while accumulated dosage and prolonged exposure to these factors results in bone destruction. Although long-term outcomes such as pituitary hormone deficiency after cranial irradiation may be irreversible, spontaneous recovery of bone mass is a privilege of this age group. BP administration, supplementation with adequate vitamin $\mathrm{D}$ and calcium, and regular exercise are beneficial options for optimal bone mineral accrual in pediatric patients with ALL. There is no definitive method for bone morbidity assessment and treatment in children with ALL. Our goal to combine up-to-date diagnostic and therapeutic options to provide appropriate screening to any patient overcoming ALL. Healthcare-givers who are responsible for treating these patients should consider bone morbidity throughout the entire disease course.

\section{Conflicts of interest}

No potential conflict of interest relevant to this article was reported.

\section{References}

1. Lee JW, Cho B. Prognostic factors and treatment of pediatric acute lymphoblastic leukemia. Korean J Pediatr 2017;60:129-37.

2. Inaba $\mathrm{H}$, Greaves M, Mullighan CG. Acute lymphoblastic leukaemia. Lancet 2013;381:1943-55.

3. Demidowicz E, Pogorzala M, Lecka M, Zolnowska H, Marjanska A, Kubicka M, et al. Outcome of pediatric acute lymphoblastic leukemia: sixty years of progress. Anticancer Res 2019;39:5203-7.

4. Sklar CA, Antal Z, Chemaitilly W, Cohen LE, Follin C, Meacham LR, et al. Hypothalamic-pituitary and growth disorders in survivors of childhood cancer: an Endocrine Society Clinical Practice Guideline. J Clin Endocrinol Metab 2018;103:2761-84.

5. Howard SC, Pui CH. Endocrine complications in pediatric patients with acute lymphoblastic leukemia. Blood Rev 2002;16:225-43

6. Mostoufi-Moab S, Ward LM. Skeletal morbidity in children and adolescents during and following cancer therapy. Horm Res Paediatr 2019;91:137-51.

7. Kizilocak H, Okcu F. Late effects of therapy in childhood acute lymphoblastic leukemia survivors. Turk J Haematol 2019;36:1-11.

8. Simm PJ, Biggin A, Zacharin MR, Rodda CP, Tham E, Siafarikas A, et al. Consensus guidelines on the use of bisphosphonate therapy in children and adolescents. J Paediatr Child Health 2018;54:223-33.

9. Mostoufi-Moab S, Halton J. Bone morbidity in childhood leukemia: epidemiology, mechanisms, diagnosis, and treatment. Curr Osteoporos Rep 2014;12:300-12.

10. Warner JT, Evans WD, Webb DK, Bell W, Gregory JW. Relative osteopenia after treatment for acute lymphoblastic leukemia. Pediatr Res 1999;45:544-51.

11. Davies JH, Evans BA, Jenney ME, Gregory JW. Skeletal morbidity in childhood acute lymphoblastic leukaemia. Clin Endocrinol (Oxf) 2005;63:1-9.

12. Shimo T, Sasaki A. Mechanism of cancer-induced bone destruction: an association of connective tissue growth factor (CTGF/CCN2) in the bone metastasis. Japanese Dental Science Review 201 1;47:13-22.

13. Mattia C, Coluzzi F, Celidonio L, Vellucci R. Bone pain mechanism in osteoporosis: a narrative review. Clin Cases Miner Bone Metab 2016;13:97-100.

14. Angsubhakorn N, Suvannasankha A. Acute lymphoblastic leukaemia with osteolytic bone lesions: diagnostic dilemma. BMJ Case Rep 2018 Aug 11;2018. pii: bcr-2018-225008. doi: 10.1136/bcr-2018-225008.

15. Shuhart CR, Yeap SS, Anderson PA, Jankowski LG, Lewiecki EM, Morse LR, et al. Executive summary of the 2019 ISCD position development conference on monitoring treatment, dxa cross-calibration and least significant change, spinal cord injury, peri-prosthetic and orthopedic bone health, transgender medicine, and pediatrics. J Clin Densitom 2019;22:453-71.

16. Cummings EA, Ma J, Fernandez CV, Halton J, Alos N, Miettunen PM, et al. Incident vertebral fractures in children with leukemia during the four years following diagnosis. J Clin Endocrinol Metab 2015;100:3408-17.

17. Halton J, Gaboury I, Grant R, Alos N, Cummings EA, Matzinger M, et al. Advanced vertebral fracture among newly diagnosed children with acute lymphoblastic leukemia: results of the Canadian Steroid-Associated Osteoporosis in the Pediatric Population (STOPP) research program. J Bone Miner Res 2009;24:1326-34.

18. Carriere B, Cummins-Mcmanus B. Vertebral fractures as initial signs for acute lymphoblastic leukemia. Pediatr Emerg Care 2001;17:258-61.

19. Stagi S, Cavalli L, Iurato C, Seminara S, Brandi ML, de Martino M. Bone health in children and adolescents: the available imaging techniques. Clin Cases Miner Bone Metab 2013;10:166-71. 
20. Genant HK, Wu CY, van Kuijk C, Nevitt MC. Vertebral fracture assessment using a semiquantitative technique. J Bone Miner Res 1993;8:1137-48.

21. Koerber F, Schulze Uphoff U, Koerber S, Schonau E, Maintz D, Semler O. Introduction of a new standardized assessment score of spine morphology in osteogenesis imperfecta. Rofo 2012;184:719-25.

22. Kim YM, Demissie S, Eisenberg R, Samelson EJ, Kiel DP, Bouxsein ML. Intra-and inter-reader reliability of semiautomated quantitative morphometry measurements and vertebral fracture assessment using lateral scout views from computed tomography. Osteoporos Int 2011;22:2677-88.

23. Alqahtani FF, Offiah AC. Diagnosis of osteoporotic vertebral fractures in children. Pediatr Radiol 2019;49:28396.

24. Levine MA. Assessing bone health in children and adolescents. Indian J Endocrinol Metab 2012;16(Suppl 2):S205-12.

25. Stagi S, Cavalli L, Cavalli T, de Martino M, Brandi ML. Peripheral quantitative computed tomography ( $\mathrm{pQCT}$ ) for the assessment of bone strength in most of bone affecting conditions in developmental age: a review. Ital J Pediatr 2016;42:88.

26. Zemel BS. Quantitative computed tomography and computed tomography in children. Curr Osteoporos Rep 2011;9:284-90.

27. Fewtrell MS, British P, Adolescent Bone G. Bone densitometry in children assessed by dual x ray absorptiometry: uses and pitfalls. Arch Dis Child 2003;88:795-8.

28. Shaw N, Crabtree N. Bone density in children: what are we measuring? Arch Dis Child 2019;104:1108-11.

29. Adams JE. Bone densitometry in children. Semin Musculoskelet Radiol 2016;20:254-68.

30. Adesina OO, Gurney JG, Kang G, Villavicencio M, Hodges JR, Chemaitilly W, et al. Height-corrected low bone density associates with severe outcomes in sickle cell disease: SCCRIP cohort study results. Blood Adv 2019;3:1476-88.

31. Lim JS, Hwang JS, Lee JA, Kim DH, Park KD, Cheon GJ, et al. Bone mineral density according to age, bone age, and pubertal stages in korean children and adolescents. J Clin Densitom 2010;13:68-76.

32. Orgel E, Mueske NM, Wren TA, Gilsanz V, Butturini AM, Freyer DR, et al. Early injury to cortical and cancellous bone from induction chemotherapy for adolescents and young adults treated for acute lymphoblastic leukemia. Bone 2016;85:131-7.

33. Pufall MA. Glucocorticoids and cancer. Adv Exp Med Biol 2015;872:315-33

34. Inaba $\mathrm{H}$, Pui CH. Glucocorticoid use in acute lymphoblastic leukaemia. Lancet Oncol 2010;11:1096-106.

35. Cushing H. The basophil adenomas of the pituitary body and their clinical manifestations (pituitary basophilism). 1932. Obes Res 1994;2:486-508.

36. Akeno N, Matsunuma A, Maeda T, Kawane T, Horiuchi N. Regulation of vitamin D-1alpha-hydroxylase and -24-hydroxylase expression by dexamethasone in mouse kidney. J Endocrinol 2000;164:339-48.

37. O'Brien CA, Jia D, Plotkin LI, Bellido T, Powers CC, Stewart SA, et al. Glucocorticoids act directly on osteoblasts and osteocytes to induce their apoptosis and reduce bone formation and strength. Endocrinology 2004;145:1835-41.

38. Jia D, O'Brien CA, Stewart SA, Manolagas SC, Weinstein RS. Glucocorticoids act directly on osteoclasts to increase their life span and reduce bone density. Endocrinology 2006;147:5592-9.

39. Tack LJ, Tatsi C, Stratakis CA, Lodish MB. Effects of glucocorticoids on bone: what we can learn from pediatric endogenous Cushing's syndrome. Horm Metab Res 2016;48:764-70.

40. Hansen KE, Kleker B, Safdar N, Bartels CM. A systematic review and meta-analysis of glucocorticoid-induced osteoporosis in children. Semin Arthritis Rheum 2014;44: 47-54.

41. Briot K, Roux C. Glucocorticoid-induced osteoporosis. RMD Open 2015;1:e000014.

42. Ward LM. Osteoporosis due to glucocorticoid use in children with chronic illness. Horm Res 2005;64:209-21.

43. Wilson CL, Ness KK. Bone mineral density deficits and fractures in survivors of childhood cancer. Curr Osteoporos Rep 2013;11:329-37.

44. Lentle B, Ma J, Jaremko JL, Siminoski K, Matzinger MA, Shenouda N, et al. The radiology of vertebral fractures in childhood osteoporosis related to glucocorticoid administration. J Clin Densitom 2016;19:81-8.

45. te Winkel ML, Pieters R, Hop WC, Roos JC, Bokkerink JP, Leeuw JA, et al. Bone mineral density at diagnosis determines fracture rate in children with acute lymphoblastic leukemia treated according to the DCOG-ALL9 protocol. Bone 2014;59:223-8.

46. Ma J, Siminoski K, Alos N, Halton J, Ho J, Cummings EA, et al. Impact of vertebral fractures and glucocorticoid exposure on height deficits in children during treatment of leukemia. J Clin Endocrinol Metab 2019;104:213-22.

47. Tian $\mathrm{H}$, Cronstein BN. Understanding the mechanisms of action of methotrexate: implications for the treatment of rheumatoid arthritis. Bull NYU Hosp Jt Dis 2007;65:16873.

48. Crofton PM, Ahmed SF, Wade JC, Elmlinger MW, Ranke $\mathrm{MB}$, Kelnar CJ, et al. Bone turnover and growth during and after continuing chemotherapy in children with acute lymphoblastic leukemia. Pediatr Res 2000;48:490-6.

49. Atkinson SA, Fraher L, Gundberg CM, Andrew M, Pai M, Barr RD. Mineral homeostasis and bone mass in children treated for acute lymphoblastic leukemia. J Pediatr 1989;114:793-800.

50. Egler RA, Ahuja SP, Matloub Y. L-asparaginase in the treatment of patients with acute lymphoblastic leukemia. J Pharmacol Pharmacother 2016;7:62-71.

51. Liu C, Janke LJ, Kawedia JD, Ramsey LB, Cai X, Mattano LA Jr, et al. Asparaginase potentiates glucocorticoidinduced osteonecrosis in a mouse model. PLoS One 2016;11:e0151433. 
52. Nissinen TA, Degerman J, Rasanen M, Poikonen AR, Koskinen S, Mervaala E, et al. Systemic blockade of ACVR2B ligands prevents chemotherapy-induced muscle wasting by restoring muscle protein synthesis without affecting oxidative capacity or atrogenes. Sci Rep 2016;6:32695.

53. Davies JH, Evans BA, Jenney ME, Gregory JW. Effects of chemotherapeutic agents on the function of primary human osteoblast-like cells derived from children. J Clin Endocrinol Metab 2003;88:6088-97.

54. Kang MJ, Lim JS. Bone mineral density deficits in childhood cancer survivors: pathophysiology, prevalence, screening, and management. Korean J Pediatr 2013;56:60-7.

55. Gilsanz V, Carlson ME, Roe TF, Ortega JA. Osteoporosis after cranial irradiation for acute lymphoblastic leukemia. The Journal of Pediatrics 1990;117:238-44.

56. Nysom K, Holm K, Michaelsen KF, Hertz H, Muller J, Molgaard C. Bone mass after treatment for acute lymphoblastic leukemia in childhood. J Clin Oncol 1998;16:375260 .

57. Kaste SC, Jones-Wallace D, Rose SR, Boyett JM, Lustig RH, Rivera GK, et al. Bone mineral decrements in survivors of childhood acute lymphoblastic leukemia: frequency of occurrence and risk factors for their development. Leukemia 2001;15:728-34.

58. Cox CL, Zhu L, Kaste SC, Srivastava K, Barnes L, Nathan PC, et al. Modifying bone mineral density, physical function, and quality of life in children with acute lymphoblastic leukemia. Pediatr Blood Cancer 2018;65:e26929.

59. Ward LM, Ma J, Lang B, Ho J, Alos N, Matzinger MA, et al. Bone morbidity and recovery in children with acute lymphoblastic leukemia: results of a six-year prospective cohort study. J Bone Miner Res 2018;33:1435-43.

60. Vitanza NA, Hogan LE, Zhang G, Parker RI. The progression of bone mineral density abnormalities after chemotherapy for childhood acute lymphoblastic leukemia. J Pediatr Hematol Oncol 2015;37:356-61.

61. Mostoufi-Moab S, Brodsky J, Isaacoff EJ, Tsampalieros A, Ginsberg JP, Zemel B, et al. Longitudinal assessment of bone density and structure in childhood survivors of acute lymphoblastic leukemia without cranial radiation. J Clin Endocrinol Metab 2012;97:3584-92.

62. von Scheven E, Corbin KJ, Stagi S, Cimaz R. Glucocorticoidassociated osteoporosis in chronic inflammatory diseases: epidemiology, mechanisms, diagnosis, and treatment. Curr Osteoporos Rep 2014;12:289-99.

63. Soyka LA, Fairfield WP, Klibanski A. Clinical review 117: Hormonal determinants and disorders of peak bone mass in children. J Clin Endocrinol Metab 2000;85:3951-63.

64. Grover M, Bachrach LK. Osteoporosis in children with chronic illnesses: diagnosis, monitoring, and treatment. Curr Osteoporos Rep 2017;15:271-82.

65. Kaste SC, Qi A, Smith K, Surprise H, Lovorn E, Boyett J, et al. Calcium and cholecalciferol supplementation provides no added benefit to nutritional counseling to improve bone mineral density in survivors of childhood acute lymphoblastic leukemia (ALL). Pediatr Blood Cancer 2014;61:885-93

66. Jayasena A, Atapattu N, Lekamwasam S. Treatment of glucocorticoid-induced low bone mineral density in children: a systematic review. Int J Rheum Dis 2015;18:28793.

67. Wagner CL, Greer FR; American Academy of Pediatrics Section on Breastfeeding; American Academy of Pediatrics Committee on Nutrition. Prevention of rickets and vitamin $\mathrm{D}$ deficiency in infants, children, and adolescents. Pediatrics 2008; 122:1142-52.

68. Greer FR, Krebs NF; American Academy of Pediatrics Committee on Nutrition. Optimizing bone health and calcium intakes of infants, children, and adolescents. Pediatrics 2006;117:578-85.

69. Sunyecz JA. The use of calcium and vitamin D in the management of osteoporosis. Ther Clin Risk Manag 2008;4:827-36

70. Saraff V, Hogler W. Endocrinology and adolescence: osteoporosis in children: diagnosis and management. Eur J Endocrinol 2015;173:R185-97.

71. Cheung MS. Drugs used in paediatric bone and calcium disorders. Endocr Dev 2015;28:277-90.

72. Simioni C, Zauli G, Martelli AM, Vitale M, Ultimo S, Milani $\mathrm{D}$, et al. Physical training interventions for children and teenagers affected by acute lymphoblastic leukemia and related treatment impairments. Oncotarget 2018;9:17199209.

73. Lucia A, Ramirez M, San Juan AF, Fleck SJ, Garcia-Castro J, Madero L. Intrahospital supervised exercise training: a complementary tool in the therapeutic armamentarium against childhood leukemia. Leukemia 2005;19:1334-7. 